Proceedings of the Edinburgh Mathematical Society (2004) 47, 411-419 (C)

DOI:10.1017/S0013091503000087 Printed in the United Kingdom

\title{
LEFT ANNIHILATORS CHARACTERIZED BY DIFFERENTIAL IDENTITIES
}

\author{
TSIU-KWEN LEE AND CHING-YUEH PAN \\ Department of Mathematics, National Taiwan University, Taipei 106, \\ Taiwan (tklee@math.ntu.edu.tw; cypan@math.ntu.edu.tw)
}

(Received 7 February 2003)

\begin{abstract}
Let $R$ be a semiprime ring with $U_{\mathrm{s}}$ its maximal symmetric ring of quotients and let $\rho_{1}$ and $\rho_{2}$ be two right ideals of $R$. We show that $\ell_{R}\left(\rho_{1}\right)=\ell_{R}\left(\rho_{2}\right)$ if and only if $\rho_{1}$ and $\rho_{2}$ satisfy the same differential identities with coefficients in $U_{\mathrm{s}}$, where $\ell_{R}\left(\rho_{i}\right)$ denotes the left annihilator of $\rho_{i}$ in $R$. This
\end{abstract} gives a generalization of several previous results in this area.

Keywords: semiprime ring; maximal ring of quotients; generalized polynomial identities (GPIs); differential identity

2000 Mathematics subject classification: Primary 6R50

Secondary 16 N60

\section{Introduction}

Throughout, $R$ always denotes a semiprime ring. A right ideal $\rho$ of $R$ is said to be dense if $\rho_{R}$ is a dense submodule of $R_{R}$. That is, given $x, y \in R$ with $y \neq 0$, there exists $r \in R$ such that $x r \in \rho$ and $y r \neq 0$. The maximal right ring of quotients (or the right Utumi quotient ring used in [8]) of $R$ can be characterized as a ring $U$ satisfying the following axioms.

(1) $R$ is a subring of $U$.

(2) For each $a \in U$, there exists a dense right ideal $\rho$ of $R$ such that $a \rho \subseteq R$.

(3) If $a \in U$ and $a \rho=0$ for some dense right ideal $\rho$ of $R$, then $a=0$.

(4) For any dense right ideal $\rho$ of $R$ and for any right $R$-module homomorphism $\phi$ : $\rho_{R} \rightarrow R_{R}$, there exists $a \in U$ such that $\phi(x)=a x$ for all $x \in \rho$.

The maximal symmetric ring of quotients $U_{\mathrm{s}}$ of $R$ is then defined as

$$
U_{\mathrm{s}}=\{x \in U \mid \lambda x \subseteq R \text { for some dense left ideal } \lambda \text { of } R\} .
$$

Then $U$ and $U_{\mathrm{s}}$ are still semiprime rings and have the same centre, denoted by $C$, which is called the extended centroid of $R$. For these basic properties we refer to [2]. The lifting 
properties of generalized polynomial identities (or GPIs for brevity) have been studied by Beidar $[\mathbf{1}]$ and by Chuang $[\mathbf{3}]$. We mention the two main results here.

Theorem 1.1 (Beidar). Let $R$ be a semiprime ring. Then $R$ and $U$ satisfy the same GPIs with coefficients in $U$.

Theorem 1.2 (Chuang). Let $R$ be a prime ring. Then $R$ and each dense right ideal of $R$ satisfy the same GPIs with coefficients in $U$.

In $[8]$, the first-named author gave another viewpoint on the two theorems above. We characterize the GPIs satisfied by two right ideals $\rho_{1}$ and $\rho_{2}$ of a semiprime ring $R$ by checking left annihilators of $\rho_{1}$ and $\rho_{2}$. For a subset $A$ of $R$ we denote by $\ell_{R}(A)$ the left annihilator of $A$ in $R$, that is, $\ell_{R}(A)=\{x \in R \mid x A=0\}$. Similarly, we define the right annihilator $r_{R}(A)$ of $A$ in $R$. Applying [8, Main Theorem] and Theorem 1.1 we have the following immediate consequence.

Theorem 1.3. Let $R$ be a semiprime ring with two right ideals $\rho_{1}$ and $\rho_{2}$. Then $\ell_{R}\left(\rho_{1}\right)=\ell_{R}\left(\rho_{2}\right)$ if and only if $\rho_{1}$ and $\rho_{2}$ satisfy the same GPIs with coefficients in $U_{\mathrm{s}}$.

This theorem says that to test whether the two right ideals $\rho_{1}$ and $\rho_{2}$ satisfy the same GPIs with coefficients in $U_{\mathrm{s}}$, it suffices to check only the simplest type $a X$ with $a \in U_{\mathrm{s}}$. In further study along this line, the first-named author [9] considered the lifting properties of differential identities (DIs) on right ideals with zero left annihilators. The structure theory of differential identities has been established by Kharchenko in $[\mathbf{6}, \mathbf{7}]$. To state these results precisely, let us recall some notation. An additive map $d: A \rightarrow U$, where $A$ is a subring of $U$, is called a derivation if $(x y)^{d}=x^{d} y+x y^{d}$ for all $x, y \in A$. We denote by $\operatorname{Der}(U)$ the set of all derivations of $U$. For $d \in \operatorname{Der}(U)$ and $x \in U$, we define $x^{d \beta}=x^{d} \beta$ for $\beta \in C$. It follows that $\operatorname{Der}(U)$ forms a right $C$-module. Let $\boldsymbol{D}$ be the $C$-submodule of $\operatorname{Der}(U)$ defined by

$$
\boldsymbol{D}=\left\{\delta \in \operatorname{Der}(U) \mid I^{\delta} \subseteq R \text { for some dense ideal } I \text {, depending on } \delta, \text { of } R\right\} .
$$

In fact, every derivation $d: I \rightarrow R$, where $I$ is a dense ideal of $R$, can be uniquely extended to a derivation of $U$ such that $U_{\mathrm{s}}^{d} \subseteq U_{\mathrm{s}}$. Thus $d \in \boldsymbol{D}$ in this case. By a derivation word we mean an additive map $\Delta$ from $U$ into itself assuming the form $\Delta=\delta_{1} \delta_{2} \cdots \delta_{t}$, where each $\delta_{i} \in \boldsymbol{D}$. If $\Delta$ is empty, we define $x^{\Delta}=x$ for $x \in U$. A differential polynomial means a generalized polynomial with coefficients in $U$ and with non-commuting variables $X_{i}$ which are acted on by derivation words. Thus every differential polynomial can be written in the form $\phi\left(X_{i}^{\Delta_{j}}\right)$, where $\phi\left(Z_{i j}\right)$ is a generalized polynomial over $U$ in distinct indeterminates $Z_{i j}$, and the $\Delta_{j}$ are derivation words. A differential polynomial $\phi\left(X_{i}^{\Delta_{j}}\right)$ is called a differential identity (DI) for a subset $T$ of $U$ if $\phi\left(X_{i}^{\Delta_{j}}\right)$ assumes 0 for any assignment of values from $T$ to its indeterminates $X_{i}$. The first-named author proved the following theorem (see $[\mathbf{9}$, Theorem 6]).

Theorem 1.4. Let $R$ be a semiprime ring and let $\rho$ be a right ideal of $R$ with zero left annihilator. Then $R$ and $\rho$ satisfy the same DIs with coefficients in $U_{\mathrm{s}}$. 
The first-named author also raised a natural generalization of Theorem 1.4 (see $[\mathbf{9}$, p. 807]). Let $\rho_{1}$ and $\rho_{2}$ be two right ideals of a semiprime ring $R$ with $\ell_{R}\left(\rho_{1}\right)=\ell_{R}\left(\rho_{2}\right)$. Do $\rho_{1}$ and $\rho_{2}$ satisfy the same DIs with coefficients in $U_{\mathrm{s}}$ ? The purpose of this paper is to give an answer in the affirmative. Our main theorem is as follows.

Theorem 1.5. Let $R$ be a semiprime ring with two right ideals $\rho_{1}$ and $\rho_{2}$. Then $\ell_{R}\left(\rho_{1}\right)=\ell_{R}\left(\rho_{2}\right)$ if and only if $\rho_{1}$ and $\rho_{2}$ satisfy the same DIs with coefficients in $U_{\mathrm{s}}$.

Theorem 1.5 implies that, to test whether the two right ideals $\rho_{1}$ and $\rho_{2}$ satisfy the same DIs with coefficients in $U_{\mathrm{s}}$, it suffices to check only the simplest type $a X$ with $a \in U_{\mathrm{s}}$. Also, Theorem 1.4 is an immediate consequence of Theorem 1.5. Indeed, if $\rho$ is a right ideal of $R$ with $\ell_{R}(\rho)=0$, then $\ell_{R}(\rho)=0=\ell_{R}(R)$. Thus, by Theorem $1.5, R$ and $\rho$ satisfy the same DIs with coefficients in $U_{\mathrm{s}}$, as asserted.

\section{The prime case}

The aim of this section is to prove the prime case of Theorem 1.5. We denote by $Q$ the symmetric Martindale quotient ring of $R$, that is

$$
Q=\{x \in U \mid I x+x I \subseteq R \text { for some dense ideal } I \text { of } R\} .
$$

We note that $U_{\mathrm{s}}=Q$ if $R$ is a prime ring with non-zero $\operatorname{socle~} \operatorname{soc}(R)$. A derivation $d \in \boldsymbol{D}$ is called $X$-inner if $d$ is an inner derivation induced by an element of $Q$. Otherwise, it is called $X$-outer. We set $D_{\text {int }}$ to be the $C$-submodule of $\operatorname{Der}(U)$ consisting of all $X$-inner derivations. Then the following hold.

(1) For $\delta, d \in \boldsymbol{D}$ we have that $[\delta, d] \in \boldsymbol{D}$ and $\delta^{p} \in \boldsymbol{D}$ if char $R=p$ is a prime integer.

(2) $D_{\text {int }} \subseteq \boldsymbol{D} \subseteq \operatorname{Der}(Q)$.

(3) If $\delta \in \boldsymbol{D}$ is $U$-inner, then $\delta$ must be $X$-inner.

Recall the following basic identities due to Kharchenko [6, p. 155].

(B1) $(X Y)^{\delta}=X^{\delta} Y+X Y^{\delta}$ for $\delta \in \boldsymbol{D}$.

(B2) $(X+Y)^{\delta}=X^{\delta}+Y^{\delta}$ for $\delta \in \boldsymbol{D}$.

(B3) $X^{\delta}=X a-a X$ if $\delta$ is the inner derivation induced by $a \in Q$.

(B4) $X^{[d, \delta]}=\left(X^{d}\right)^{\delta}-\left(X^{\delta}\right)^{d}$ for $d, \delta \in \boldsymbol{D}$.

(B5) $X^{\delta^{p}}=\left(\cdots\left(\left(X^{\delta}\right)^{\delta}\right) \cdots\right)^{\delta}$ (p-times) for $\delta \in \boldsymbol{D}$ and char $R=p>0$. If char $R=0$, then this identity assumes the form $X=X$.

(B6) $X^{d \alpha+\delta \beta}=\alpha X^{d}+\beta X^{\delta}$ for $d, \delta \in \boldsymbol{D}$ and $\alpha, \beta \in C$. 
Let $R$ always be a prime ring from now on. Then $C$ is a field. We choose a fixed basis $M_{0}$ for $D_{\text {int }}$ and augment it to a basis $M$ for $\boldsymbol{D}$ over $C$. Fix a total order ' $>$ ' in the set $M$ such that $\mu_{0}>\mu$ for $\mu_{0} \in M_{0}$ and $\mu \in M \backslash M_{0}$, and then extend this order to the set of all derivation words by assuming that a longer word is greater than a shorter one and that words of the same length are ordered lexicographically. A regular word means a derivation word of the form $\Delta=\delta_{1}^{s_{1}} \delta_{2}^{s_{2}} \cdots \delta_{m}^{s_{m}}$ possessing the following properties:

(W1) $\delta_{i} \in M \backslash M_{0}$ for $1 \leqslant i \leqslant m$;

(W2) $\delta_{1}<\delta_{2}<\cdots<\delta_{m}$; and

(W3) $s_{i}<p$ for $1 \leqslant i \leqslant m$, if $\operatorname{char} R=p>0$.

As pointed out in $[\mathbf{6}, \mathbf{7}]$, each differential identity can be transformed, via the basic identities (B1)-(B6), into a form $\phi\left(X_{i}^{\Delta_{j}}\right)$ such that

(R1) $\phi\left(Z_{i j}\right)$ is a generalized polynomial with coefficients in $U$ in non-commuting indeterminates $Z_{i j}$; and

(R2) the $\Delta_{j}$ are distinct regular words.

A differential polynomial is called reduced if it assumes the form $\phi\left(X_{i}^{\Delta_{j}}\right)$ satisfying (R1) and (R2). Kharchenko actually proved the following powerful result [7, Theorem 2].

Theorem 2.1 (Kharchenko's Theorem). Let $R$ be a prime ring. If $\phi\left(X_{i}^{\Delta_{j}}\right)$ is a reduced DI (with coefficients in $U$ ) for a non-zero ideal of $R$, then $\phi\left(Z_{i j}\right)$ is a GPI for $R$.

Since every differential polynomial can be transformed into a reduced differential polynomial via (B1)-(B6) and $\rho R \subseteq \rho \subseteq \rho C \subseteq \rho U$, applying Theorems 2.1, 1.1 and 1.2 we have the following corollary.

Corollary 2.2. Let $R$ be a prime ring with extended centroid $C$ and let $\rho$ be a right ideal of $R$. Then $\rho U, \rho C$ and $\rho$ satisfy the same DIs with coefficients in $U$.

Corollary 2.3. Let $R$ be a prime ring with $I$ a non-zero ideal of $R$. Then $I$ and $U$ satisfy the same DIs with coefficients in $U$.

Let $B$ be a set of $C$-independent elements of $U_{\mathrm{s}}$ and let $\Delta_{1}, \Delta_{2}, \ldots, \Delta_{t}$ be distinct regular words. A $B$-monomial in $X^{\Delta_{i}}$ means a monomial of the form $u_{0} Y_{1} u_{1} Y_{2} \cdots Y_{n} u_{n}$, where $u_{i} \in B$ and $Y_{i} \in\left\{X^{\Delta_{1}}, X^{\Delta_{2}}, \ldots, X^{\Delta_{t}}\right\}$ for each $i$. Here, each $u_{i} Y_{i+1}$ is called a submonomial appeared in this monomial. Thus for each non-zero $\phi \in U_{\mathrm{s}} *_{C}$ $C\left\{X^{\Delta_{1}}, X^{\Delta_{2}}, \ldots, X^{\Delta_{t}}\right\}$, the free product of the $C$-algebra $U_{\mathrm{s}}$ and the free $C$-algebra $C\left\{X^{\Delta_{1}}, X^{\Delta_{2}}, \ldots, X^{\Delta_{t}}\right\}$, there exists a $B$ such that $\phi$ is a $C$-linear combination of $B$-monomials in $X^{\Delta_{i}}$. Also, $B$ is said to be $C$-independent modulo $\ell_{U_{\mathrm{s}}}(\rho)$, where $\rho$ is a right ideal of $R$, if $B$ satisfies the following condition: if $\beta_{1}, \ldots, \beta_{\ell} \in C$ satisfy $\left(\beta_{1} b_{1}+\cdots+\beta_{\ell} b_{\ell}\right) \rho=0$, where these $b_{i}$ are distinct elements in $B$, then $\beta_{i}=0$ for all $i$. We begin our proof with the following result [8, Lemma 3]. 
Lemma 2.4. Let $\rho$ be a non-zero right ideal of $R$. Suppose that $a_{1}, a_{2}, \ldots, a_{t} \in U_{\mathrm{s}}$ are $C$-independent modulo $\ell_{U_{\mathrm{s}}}(\rho)$. Then there exists an element $u \in \rho$ such that $a_{1} u, \ldots, a_{t} u$ are $C$-independent unless $R$ is a PI-ring.

We write $U_{\mathrm{s}}=\ell_{U_{\mathrm{s}}}(\rho) \oplus W_{\rho}$ as $C$-spaces and fix a basis $B_{\rho}$ for $W_{\rho}$. Thus $B_{\rho}$ is $C$ independent modulo $\ell_{U_{\mathrm{s}}}(\rho)$.

Lemma 2.5. Let $\rho$ be a right ideal of $R$ and let $\Delta$ be a non-empty regular word. If $a \in \ell_{U_{\mathrm{s}}}(\rho)$, then there exist finitely many regular words $G_{n}<G_{n-1}<\cdots<G_{1}<\Delta$ such that

$$
a x^{\Delta}=\sum_{i=1}^{n}\left(\sum_{j=1}^{m_{i}} \beta_{i j} b_{i j}\right) x^{G_{i}}
$$

for all $x \in r_{R}\left(\ell_{R}(\rho)\right)$, where, for each $i$, the set $\left\{b_{i j} \mid j=1, \ldots, m_{i}\right\}$ is $C$-independent in $B_{\rho}$, and $\beta_{i j} \in C$.

Proof. Since $a \in \ell_{U_{\mathrm{s}}}(\rho)$, we have $a \rho=0$ and hence $a x=0$ for all $x \in r_{R}\left(\ell_{R}(\rho)\right)$. Let $x \in r_{R}\left(\ell_{R}(\rho)\right)$. Then

$$
0=(a x)^{\Delta}=a x^{\Delta}+\sum_{i>1} a^{E_{i}} x^{F_{i}}
$$

where these $\left(E_{i}, F_{i}\right)$ run over all pairs of subwords of $\Delta$ with $E_{1}=\emptyset, F_{1}=\Delta$. Note that $a^{E_{i}} \in U_{\mathrm{s}}$ since $U_{\mathrm{s}}^{E_{i}} \subseteq U_{\mathrm{s}}$. By the fact that $U_{\mathrm{s}}=\ell_{U_{\mathrm{s}}}(\rho) \oplus B_{\rho} C$, we can write

$$
a x^{\Delta}=\sum_{i>1} \sum_{j} \mu_{i j} c_{i j} x^{F_{i}}+\sum_{i>1} c_{i} x^{F_{i}}
$$

where $c_{i j} \in B_{\rho}, c_{i} \in \ell_{U_{\mathrm{s}}}(\rho)$ and $\mu_{i j} \in C$. Since $c_{i} \in \ell_{U_{\mathrm{s}}}(\rho)$ and $F_{i}<\Delta$ for $i>1$, we can repeat the same argument on $c_{i} x^{F_{i}}$ and get our conclusion by the inductive hypothesis. This proves the lemma.

We are now in a position to prove the following key lemma, which reduces Theorem 1.5 to the prime GPI case.

Lemma 2.6. Let $\phi\left(X^{\Delta_{i}}\right)$ be a reduced DI for a right ideal $\rho$ of $R$. Then either $R$ is a GPI-ring or $\phi\left(X^{\Delta_{i}}\right)$ is a reduced DI for $r_{R}\left(\ell_{R}(\rho)\right)$.

Proof. Choose $B_{\rho}^{\prime}$ to be a $C$-basis for $\ell_{U_{\mathrm{s}}}(\rho)$. Since $U_{\mathrm{s}}=\ell_{U_{\mathrm{s}}}(\rho) \oplus B_{\rho} C=B_{\rho}^{\prime} C \oplus B_{\rho} C$, we can write

$$
\phi\left(X^{\Delta_{i}}\right)=\phi_{0}\left(X^{\Delta_{i}}\right)+\phi_{1}\left(X^{\Delta_{i}}\right),
$$

where $\phi_{0}\left(X^{\Delta_{i}}\right)$ is a $C$-linear combination of $B_{\rho}$-monomials in $X^{\Delta_{i}}$ and where each monomial of $\phi_{1}\left(X^{\Delta_{i}}\right)$ has coefficients in $B_{\rho} \cup B_{\rho}^{\prime}$ and has at least a coefficient in $B_{\rho}^{\prime}$. Applying (2.1) to all possible submonomials $a X^{\Delta_{i}}$, where $a \in \ell_{U_{\mathrm{s}}}(\rho)$, of $\phi_{1}\left(X^{\Delta_{i}}\right)$, we can transform $\phi\left(X^{\Delta_{i}}\right)$ to a differential polynomial $\psi\left(X^{\Gamma_{j}}\right)$, where $\psi\left(X^{\Gamma_{j}}\right)$ is a $C$-linear combination of $B_{\rho}$-monomials in $X^{\Gamma_{j}}$ or $B_{\rho^{-}}$-monomials in $X^{\Gamma_{j}}$ with their last right coefficients in $B_{\rho}^{\prime}$, where these $\Gamma_{j}$ are distinct regular words. That is,

$$
\psi\left(X^{\Gamma_{j}}\right)=\psi_{0}\left(X^{\Gamma_{j}}\right)+\psi_{1}\left(X^{\Gamma_{j}}\right)
$$


where $\psi_{0}\left(X^{\Gamma_{j}}\right)$ is a $C$-linear combination of $B_{\rho}$-monomials in $X^{\Gamma_{j}}$ and each monomial of $\psi_{1}\left(X^{\Gamma_{j}}\right)$ has the form $u_{0} Y_{1} u_{1} Y_{2} \cdots Y_{n} u_{n}$, where $u_{i} \in B_{\rho}$ for each $1 \leqslant i \leqslant n-1$ and $u_{n} \in B_{\rho}^{\prime}$ and $Y_{i} \in\left\{X^{\Gamma_{1}}, X^{\Gamma_{2}}, \ldots\right\}$ for each $i$. We may assume that $\Gamma_{j} \neq \emptyset$ for some $j$. Otherwise, we are done by Theorem 1.3.

Case 1. Suppose that $\psi\left(Z_{j}\right)$ is zero as a generalized polynomial in indeterminates $Z_{j}$. Then, in particular, $\psi\left(x^{\Gamma_{j}}\right)=0$ for all $x \in r_{R}\left(\ell_{R}(\rho)\right)$. Applying (2.1) to reverse the process from $\psi\left(X^{\Gamma_{j}}\right)$ to $\phi\left(X^{\Delta_{i}}\right)$, we conclude that $\phi\left(X^{\Delta_{i}}\right)$ is a DI for $r_{R}\left(\ell_{R}(\rho)\right)$, as asserted.

Case 2. Suppose that $\psi\left(Z_{j}\right)$ is not zero as a generalized polynomial in indeterminates $Z_{j}$. We claim that $R$ is a prime GPI-ring. Suppose not. In particular, $R$ is not a PI-ring. We list all coefficients appearing in $\psi\left(Z_{j}\right)$ as $b_{1}, b_{2}, \ldots, b_{m} \in B_{\rho}$ and $b_{1}^{\prime}, b_{2}^{\prime}, \ldots, b_{m^{\prime}}^{\prime} \in B_{\rho}^{\prime}$. Then these elements $b_{s}$ are $C$-independent modulo $\ell_{U_{\mathrm{s}}}(\rho)$. By Lemma 2.4, there exists an element $u \in \rho$ such that $b_{1} u, \ldots, b_{m} u$ are $C$-independent. Since $\psi\left(X^{\Gamma_{j}}\right)$ is a DI for $\rho, \psi\left((u X)^{\Gamma_{j}}\right)$ is a DI for $R$. Note that

$$
b_{s}(u X)^{\Gamma_{j}}=\left(b_{s} u\right) X^{\Gamma_{j}}+\sum_{l} b_{s} u^{A_{j l}} X^{B_{j l}},
$$

where the $\left(A_{j l}, B_{j l}\right)$ are pairs of subwords of $\Gamma_{j}$ with $B_{j l}<\Gamma_{j}$. Thus, by $(2.2)$, we can write

$$
\psi\left((u X)^{\Gamma_{j}}\right)=g\left(X^{\Gamma_{j}}\right)+h\left(X^{\Gamma_{j}}, X^{D_{k}}\right),
$$

where $g\left(X^{\Gamma_{j}}\right)$ is the differential polynomial obtained from $\psi\left(X^{\Gamma_{j}}\right)$ by replacing all coefficients $b_{s}$ by $b_{s} u$ and where each $D_{k}$ is a subword of some $\Gamma_{j}$ and does not appear in $\left\{\Gamma_{1}, \Gamma_{2}, \ldots\right\}$. Applying Kharchenko's Theorem to $(2.3), g\left(X_{j}\right)+h\left(X_{j}, Z_{k}\right)$ is a GPI for $R$. This GPI is indeed non-trivial since the largest monomial (ordered by considering these $\Gamma_{j}$ appearing in this monomial plus their weights and the $C$-independence of these $b_{s} u$ and $\left.b_{s^{\prime}}^{\prime}\right)$ of $g\left(X^{\Gamma_{j}}\right)$ cannot be cancelled by the terms in $h\left(X^{\Gamma_{j}}, X^{D_{k}}\right)$, a contradiction. Thus $R$ is a prime GPI-ring, proving the lemma.

With Lemma 2.6 in hand we turn our attention to the prime GPI case. The key to this case is to study the continuity of derivations in certain endomorphism rings under the finite topology. We need to recall some notation from $[\mathbf{5}$, p. 27]. Let $\Gamma$ be a ring and let $\Gamma_{\Gamma} M_{1}$ and ${ }_{\Gamma} M_{2}$ be left $\Gamma$-modules. For $S \subseteq \operatorname{Hom}_{\Gamma}\left(M_{1}, M_{2}\right)$, we let

$$
S^{\perp}=\left\{m \in M_{1} \mid m s=0 \forall s \in S\right\},
$$

and for $N \subseteq M_{1}$, we let

$$
N^{\perp}=\left\{g \in \operatorname{Hom}_{\Gamma}\left(M_{1}, M_{2}\right) \mid n g=0 \forall n \in N\right\} .
$$

First we quote the following result [5, Theorem 1].

Theorem 2.7. Let $M_{1}$ and $M_{2}$ be left vector spaces over a division ring $\Gamma$, let $A$ be a subring of $\operatorname{End}\left({ }_{\Gamma} M_{2}\right)$ and let $(B,+, 0)$ be an additive subgroup of $\operatorname{Hom}_{\Gamma}\left(M_{1}, M_{2}\right)$ such that $B A \subseteq B$. Suppose that (i) $\left(M_{2}\right)_{A}$ is irreducible and (ii) $\Gamma=\operatorname{End}\left(\left(M_{2}\right)_{A}\right)$. Then $\left(B^{\perp}\right)^{\perp}=\operatorname{cl}(B)$, the closure of $B$ in the finite topology of $\operatorname{Hom}_{\Gamma}\left(M_{1}, M_{2}\right)$. 
We now apply the theorem to our case. Let $R$ be a prime ring with non-zero socle $\operatorname{soc}(R)$. We choose a minimal idempotent $e \in R$ and let $D=e R e$ and $V=e R$. By the Density Theorem [4, Theorem 2.1.2], and considering right multiplication, we can regard $R$ as a dense subring in $\operatorname{End}\left({ }_{D} V\right)$ under its finite topology $\mathcal{T}_{R}$. For a finitedimensional $D$-subspace $W$ of ${ }_{D} V$, we define $W^{\perp}=\left\{f \in \operatorname{End}\left({ }_{D} V\right) \mid w f=0 \forall w \in W\right\}$. We recall that $\mathcal{T}_{R}$ has these $W^{\perp}+g$, where $g \in \operatorname{End}\left({ }_{D} V\right)$, as its subbasis. Since $\operatorname{soc}(R)=$ $R e R, e R q \subseteq e R$ for $q \in Q$. Thus $V$ forms a right $Q$-module and $D=\operatorname{End}\left(V_{Q}\right)$. So $Q$ is also embedded in $\operatorname{End}\left({ }_{D} V\right)$. We will make these assumptions in Theorems 2.8 and 2.9

Theorem 2.8. Let $\rho$ be a right ideal of $R$. Then $\operatorname{cl}(\rho) \cap R=r_{R}\left(\ell_{R}(\rho)\right)$.

Proof. Since $\rho R \subseteq \rho$ and $D=\operatorname{End}\left(V_{R}\right)$, by Theorem 2.7 we have $\left(\rho^{\perp}\right)^{\perp}=\operatorname{cl}(\rho)$ in $\operatorname{End}\left({ }_{D} V\right)$. We first notice that

$$
\rho^{\perp}=\{e r \in V \mid \operatorname{er} \rho=0\}=e R \cap \ell_{R}(\rho) .
$$

Also, $y \in \operatorname{cl}(\rho) \cap R=\left(\rho^{\perp}\right)^{\perp} \cap R$ if and only if $\left(e R \cap \ell_{R}(\rho)\right) y=0$. Thus it suffices to prove that if $y \in R$, then $\left(e R \cap \ell_{R}(\rho)\right) y=0$ if and only if $y \in r_{R}\left(\ell_{R}(\rho)\right)$. The 'if' part is trivial. Let $y \in R$ be such that $\left(e R \cap \ell_{R}(\rho)\right) y=0$. Then, for $u \in \ell_{R}(\rho), e R u \in e R \cap \ell_{R}(\rho)$ and so $e R u y=0$, implying that $u y=0$ by the primeness of $R$. So $\ell_{R}(\rho) y=0$ and hence $y \in r_{R}\left(\ell_{R}(\rho)\right)$, proving the theorem.

Theorem 2.9. Suppose that $d: A \rightarrow Q$ is a derivation, where $A$ is a subring of $R$ containing the socle of $R$. Then $d$ is a continuous map.

Proof. Let ${ }_{D} L$ be a finite-dimensional $D$-subspace of ${ }_{D} V$. It suffices to find a finitedimensional $D$-subspace $W$ of ${ }_{D} V$ such that $\left(W^{\perp} \cap A\right)^{d} \subseteq L^{\perp}$. We write $L=D u_{1} \oplus$ $\cdots \oplus D u_{s}$, where $u_{i} \in V$, and let $W=L+\sum_{i=1}^{s} D e u_{i}^{d}$.

Let $a \in W^{\perp} \cap A$, then $u_{i} a=0=e u_{i}^{d} a$. Since $u_{i} \in e R \subseteq \operatorname{soc}(R) \subseteq A$, we have $\left(u_{i} a\right)^{d}=0$ and so $u_{i}^{d} a+u_{i} a^{d}=0$. Thus $u_{i} a^{d}=-u_{i}^{d} a$ and so $u_{i} a^{d}=e u_{i} a^{d}=-e u_{i}^{d} a=0$. This implies that $a^{d} \in L^{\perp}$. The theorem is thus proved.

As an immediate consequence of Theorem 2.9, we have the following corollary.

Corollary 2.10. Suppose that $d: I \rightarrow Q$ is a derivation, where $I$ is an ideal of $R$. Then $d$ is a continuous map.

We are now ready to prove the main result in this section.

Theorem 2.11. Let $R$ be a prime ring with two right ideals $\rho_{1}$ and $\rho_{2}$. Then $\ell_{R}\left(\rho_{1}\right)=$ $\ell_{R}\left(\rho_{2}\right)$ if and only if $\rho_{1}$ and $\rho_{2}$ satisfy the same DIs with coefficients in $U_{\mathrm{s}}$.

Proof. The 'if' part is trivial. We prove the 'only if' part. Suppose that $\phi\left(X_{i}^{\Delta_{j}}\right)$ is a DI for a right ideal $\rho_{1}$ of $R$. Since $\ell_{R}\left(\rho_{1}\right)=\ell_{R}\left(\rho_{2}\right)$, we have $r_{R}\left(\ell_{R}\left(\rho_{1}\right)\right)=r_{R}\left(\ell_{R}\left(\rho_{2}\right)\right)$ and $\rho_{2} \subseteq r_{R}\left(\ell_{R}\left(\rho_{2}\right)\right)$. Thus it suffices to prove that $\phi\left(X_{i}^{\Delta_{j}}\right)$ is a DI for $r_{R}\left(\ell_{R}\left(\rho_{1}\right)\right)$. For simplicity of notation, we set $\rho=\rho_{1}$. Since every DI can be transformed into a reduced DI via (B1)-(B6), we may assume that $\phi\left(X_{i}^{\Delta_{j}}\right)$ is a reduced DI for $\rho$. Moreover, by assigning $X_{2}, X_{3}, \ldots$ to fixed elements in $\rho$ we may assume that $\phi$ only involves one 
indeterminate $X$ with coefficients in $U_{\mathrm{s}}$. Write $\phi=\phi\left(X^{\Delta_{j}}\right)$, where the $\Delta_{1}, \ldots, \Delta_{t}$ are all distinct regular words occurring in $\phi$.

In view of Lemma 2.6, either $R$ is a GPI-ring or $\phi\left(X^{\Delta_{j}}\right)$ is a reduced DI for $r_{R}\left(\ell_{R}(\rho)\right)$. Thus it suffices to consider the case in which $R$ is a prime GPI-ring. By Martindale's Theorem [10, Theorem 3], $R C$ is a primitive ring with a minimal right ideal $e R C$, where $e$ is a minimal idempotent in $R C$. We let $D=e R C e$ and $V=e R C$, a left vector space over the division ring $D$. Denote by $H$ the socle of $R C$. By the Density Theorem $[4$, Theorem 2.1.2], $R C$ is canonically embedded in $\operatorname{End}\left({ }_{D} V\right)$ as a dense subring. Let $d \in \boldsymbol{D}$. Then, by definition, there exists a non-zero ideal $I$ of $R$ such that $I^{d} \subseteq R$. It is clear that $H \subseteq I C$ and $H=H^{2}$. Thus we always have $H^{d} \subseteq H$. Also, $U_{\mathrm{s}}$ is canonically embedded in $\operatorname{End}\left({ }_{D} V\right)$. Theorem 2.9 says that $d: H \rightarrow H$ is a continuous map. This means that the map $x \in H \mapsto \phi\left(x^{\Delta_{j}}\right) \in \operatorname{End}\left({ }_{D} V\right)$ defines a continuous map from $H$ into $\operatorname{End}\left({ }_{D} V\right)$. Since $\rho H \subseteq \rho R C \subseteq \rho C$, applying Corollary 2.2 we have $\phi\left(x^{\Delta_{j}}\right)=0$ for all $x \in \rho H$. By the continuity of the map $x \in H \mapsto \phi\left(x^{\Delta_{j}}\right) \in \operatorname{End}\left({ }_{D} V\right)$ and Theorem 2.8, we have $\phi\left(x^{\Delta_{j}}\right)=0$ for all $x \in r_{H}\left(\ell_{H}(\rho H)\right)$.

Let $a \in r_{R}\left(\ell_{R}(\rho)\right)$. Then $\ell_{R}(\rho) a=0$ and so $\ell_{R C}(\rho H) a=0$. In particular, $\ell_{H}(\rho H) a=0$. Thus $a H \subseteq r_{H}\left(\ell_{H}(\rho H)\right)$. Hence, $r_{R}\left(\ell_{R}(\rho)\right) H \subseteq r_{H}\left(\ell_{H}(\rho H)\right)$ follows. Thus $\phi\left(x^{\Delta_{j}}\right)=0$ for all $x \in r_{R}\left(\ell_{R}(\rho)\right) H$. Since $H$ and $U$ satisfy the same DIs by Corollary 2.3, so do $r_{R}\left(\ell_{R}(\rho)\right) H$ and $r_{R}\left(\ell_{R}(\rho)\right) U$. In particular, $\phi\left(x^{\Delta_{j}}\right)=0$ for all $x \in r_{R}\left(\ell_{R}(\rho)\right)$, proving the theorem.

\section{Proof of the Main Theorem}

Let $R$ be a semiprime ring. Recall that a subset $T \subseteq U$ is called orthogonally complete if $0 \in T$ and, given any set of orthogonal idempotents $\left\{e_{\omega} \mid \omega \in \Omega\right\} \subseteq C$ and any subset $\left\{x_{\omega} \mid \omega \in \Omega\right\} \subseteq T$, there exists $x \in T$ such that $e_{\omega} x=e_{\omega} x_{\omega}$ for all $\omega \in \Omega$. For any subset $K \subseteq U$, denote by $\hat{K}$ the orthogonal completion of $K$ in $U$ which is defined as the intersection of all orthogonally complete subsets of $U$ containing $K$. Note that $\hat{K}$ itself is an orthogonally complete subset of $U$. We now come to the proof of Theorem 1.5. Since the method of extending Theorem 2.11 to the semiprime case is almost routine by applying the theory of orthogonal completions for semiprime rings [2, Chapter 3], we only sketch its proof.

Proof of Theorem 1.5. The 'if' part is trivial. We prove the 'only if' part. Suppose that $\ell_{R}\left(\rho_{1}\right)=\ell_{R}\left(\rho_{2}\right)$, where $\rho_{1}$ and $\rho_{2}$ are right ideals of $R$. Suppose that $\phi\left(X_{i}^{\Delta_{j}}\right)$ (with coefficients in $U_{\mathrm{s}}$ ) is a DI for $\rho_{1}$. Then, applying the same argument as that of $[\mathbf{8}$, Lemma 6(i)] (with DIs instead of GPIs), we see that $\phi\left(X_{i}^{\Delta_{j}}\right)$ is still a DI for $\hat{\rho}_{1}$. Let $P$ be a maximal ideal of $C$. Then the following hold:

(i) $P U$ is a prime ideal of $U$;

(ii) $\left(U_{\mathrm{s}}+P U\right) / P U$ is contained in the maximal symmetric ring of quotients of the prime ring $(\hat{R}+P U) / P U$;

(iii) $\ell_{(\hat{R}+P U) / P U}\left(\left(\hat{\rho}_{1}+P U\right) / P U\right)=\ell_{(\hat{R}+P U) / P U}\left(\left(\hat{\rho}_{2}+P U\right) / P U\right)$; and 
(iv) each $\delta \in \boldsymbol{D}$ naturally induces a derivation $\bar{\delta}$ of $U / P U$ such that $\delta(\bar{I}) \subseteq(\hat{R}+$ $P U) / P U$ for some non-zero ideal $\bar{I}$ of $(\hat{R}+P U) / P U$.

We remark that (i) is referred to in [2, Theorem 3.2.7]. For (ii), see [2, Theorem 3.2.15], and fact (iii) can be derived from the fact that $\ell_{R}\left(\rho_{1}\right)=\ell_{R}\left(\rho_{2}\right)$, Finally, fact (iv) is clear. Using these facts we can reduce the theorem to the prime case and hence $\phi\left(x_{i}^{\Delta_{j}}\right) \in P U$ for all $x_{i} \in \hat{\rho}_{2}$. Applying the fact that $\bigcap_{P} P U=0$, where the $P$ run over all maximal ideals of $C$, we see that $\phi\left(X_{i}^{\Delta_{j}}\right)$ is a DI for $\hat{\rho}_{2}$ and, therefore, for $\rho_{2}$. This proves Theorem 1.5.

\section{References}

1. K. I. BeIdAR, Rings with generalized identities, III, Vestnik Moskov. Univ. Ser. I 33 (1978), 66-73.

2. K. I. Beidar, W. S. Martindale III and A. V. Mikhalev, Rings with generalized identities (Marcel Dekker, 1996).

3. C.-L. Chunang, GPIs having coefficients in Utumi quotient rings, Proc. Am. Math. Soc. 103 (1988), 723-728.

4. I. N. Herstein, Noncommutative rings, Carus Mathematics Monograph, vol. 15 (The Mathematical Association of America, Providence, RI, 1968).

5. N. Jacobson, Structure of rings, American Mathematical Society Colloquium Publications, vol. 37, 2nd edn (American Mathematical Society, Providence, RI, 1964).

6. V. K. Kharchenko, Differential identities of prime rings, Alg. Logika 17 (1978), 220-238 (in Russian) (English transl.: Alg. Logic 17 (1978), 154-168).

7. V. K. Kharchenko, Differential identities of semiprime rings, Alg. Logika 18 (1979), 86-119 (in Russian) (English transl.: Alg. Logic 18 (1979), 58-80).

8. T.-K. LEe, Left annihilators characterized by GPIs, Trans. Am. Math. Soc. 347 (1995), 3159-3165.

9. T.-K. LEE, Differential identities of Lie ideals or large right ideals in prime rings, Commun. Alg. 27 (1999), 793-810.

10. W. S. Martindale III, Prime rings satisfying a generalized polynomial identity, J. Alg. 12 (1969), 576-584. 